\title{
The NICOLA questionnaire trial (NICOLA-QT): a randomised trial of the effect of timing and mode of delivery on the completion and return of a self-assessment questionnaire
}

\author{
Lisa Maguire ${ }^{1,2^{*}}$, Frances Burns', Mike Clarke ${ }^{1}$ \\ From 3rd International Clinical Trials Methodology Conference \\ Glasgow, UK. 16-17 November 2015
}

\section{Background}

NICOLA-QT is designed to detect the optimal time and delivery method for giving a self-assessment questionnaire to participants, seeking to overcome the low response rates associated with these. The outline has been registered as SWAT-2. NICOLA-QT is embedded in the NICOLA cohort study, which is recruiting 8,500 people over 50 years of age in Northern Ireland and follow them into old age, making a comprehensive assessment of their health, lifestyles and their decision making.

\section{Aim}

The overall aim of NICOLA-QT is to examine the impact of differing times and formats of a self-assessment questionnaire on completion rates.

\section{Methods}

Participants were randomly allocated to receive a questionnaire by either: being handed it in person at the end of a home visit or in the post approximately one week after their home visit, along with a thank-you certificate for participation to-date.

\section{Results}

The primary outcome measure is whether or not the selfcompletion questionnaire is returned. Secondary outcome measures include the time taken to return the questionnaire and the completeness of the questionnaire. Recruitment for the NICOLA cohort began in January 2014 and is expected to continue until late 2015.
Although the cohort is still recruiting, this presentation will detail preliminary findings from the interim analysis of 4000 participants in NICOLA-QT.

\section{Conclusion}

This SWAT (Study Within A Trial) aims to provide evidence on what research participants might prefer when asked to complete a questionnaire, specifically examining low-cost adjustments that researchers can use to improve return rates.

Trial Registration

NCT01978522.

Authors' details

'Queen's University Belfast, Belfast, UK. 'University of Liverpool, Liverpool, UK.

Published: 16 November 2015

doi:10.1186/1745-6215-16-S2-P107

Cite this article as: Maguire et al:: The NICOLA questionnaire trial (NICOLA-QT): a randomised trial of the effect of timing and mode of delivery on the completion and return of a self-assessment questionnaire. Trials 2015 16(Suppl 2):P107.

${ }^{1}$ Queen's University Belfast, Belfast, UK

Full list of author information is available at the end of the article 